\title{
Error-Diffused Image Security Improving Using Overall Minimal-Error Searching
}

\author{
Jing-Ming Guo* and Yun-Fu Liu \\ Department of Electrical Engineering, \\ National Taiwan University of Science and Technology \\ Taipei, Taiwan \\ jmguo@seed.net.tw, yunfuliu@gmail.com
}

\begin{abstract}
This study presents a high capacity data hiding method for generating high quality watermarked halftone images. The embedded watermarks can be distributed into single or multiple halftone images with the proposed Overall Minimal-Error Searching (OMES). The proposed method modifies the halftone values at same position of all host images with the trained Substitution Table (S-Table). The S-Table makes the original combination of these halftone values as another meaningful combination for embedded watermark, which is the key part in determining the image quality. Hence, an optimization procedure is proposed to achieve the optimized S-Table. As demonstrated in the experimental results, the proposed approach provides good image quality and is able to guard against some frequent happened attacks in printing applications.
\end{abstract}

Keywords: Digital watermarking, digital halftoning, error diffusion, iteration-based halftoning, ordered dithering, overall minimal-error searching.

\section{Introduction}

Digital halftoning [1] is a technique for changing grayscale images into two-tone halftone images, which include Ordered Dithering (OD) [1], Error Diffusion (EDF) [2], and Dot Diffusion (DDF) [3]. Among these, error diffusion offers good visual quality and reasonable computational complexity.

Digital watermarks have many usages, including protecting ownership of an image, preventing the illegal use of an image without permission, and authenticating an image to verify that it has not been altered. Currently, many methods that use halftones to embed watermarks have been studied. These techniques can be used for printing security documents such as an ID cards, currency, and confidential documents, and prevent illegal duplication and forgery by further scanning these documents to digital forms.

The watermark which is embedded in halftone images can be retrieved by scanning and applying some extraction algorithms. These methods include using a number of different dither cells to create a threshold pattern in the halftoning process [4]; using Smart Pair-Toggling (DHSPT) to embed data into error-diffused images [5];

\footnotetext{
* Member IEEE.
} 
coordinating the $\mathrm{BCH}$ error-correcting code with data-hiding techniques [6]; authentication based on halftoning and coordinate projection [7], and data hiding in several halftone images or color planes using Minimal-Error Bit Searching (MEBS) [8]. The MEBS preserves excellent image quality when embedded capacity is as high as $33.33 \%$. However, the quality degrades significantly when capacity increases up to $50 \%$. This study presents an Overall Minimal-Error Searching (OMES) to improve the image quality when the data capacity is $50 \%$.

\section{Performance Evaluations}

In this section, the performance evaluations, Peak Signal-to-Noise Ratio (PSNR) and Correct Decoding Rate (CDR), employed in this work are defined. Suppose the host image is of size $P \times Q$. The quality evaluation of Watermarked Halftone image $(W H)$ is defined as follows,

$$
P S N R=10 \log _{10} \frac{P \times Q \times 255^{2}}{\sum_{i=1}^{P} \sum_{j=1}^{Q}\left[\sum_{m, n \in R} \sum c_{m, n}\left(\text { ori }_{i+m, j+n}-w h_{i+m, j+n}\right)\right]^{2}},
$$

where the variables $\operatorname{ori}_{i, j}$ and $w h_{i, j}$ denote the grayscale value and watermarked halftone value at position $(i, j)$, respectively. The variable $c_{m, n}$ denotes the Least-Mean-Square (LMS) trained filter $(R)$ coefficient at position $(m, n)$. In this work, the size of the support region $R$ is fixed at $7 \times 7$. The LMS-trained filter can be obtained by psychophysical experiments [9]. The other way to derive the filter can use a training set of both pairs of grayscale images and good halftone results of them, such as using error diffusion or ordered dithering to produce the set. The LMS is described as follows,

$$
\begin{gathered}
\hat{h}_{i, j}=\sum_{m, n \in R} \sum c_{m, n} h_{i+m, j+n}, \\
e_{i, j}^{2}=\left(o r i_{i, j}-\hat{h}_{i, j}\right)^{2}, \\
\frac{\partial e_{i, j}^{2}}{\partial c_{m, n}}=-2 e_{i, j} h_{i+m, j+n}, \\
\left\{\begin{array}{l}
c_{m, n} \text { should be decreased, if } c_{m, n}>c_{m, n, o p t} \\
c_{m, n} \text { should be increased, if } c_{m, n}<c_{m, n, o p t} \\
c_{m, n}^{(k+1)}=c_{m, n}^{k}+\mu e_{i+m, j+n} h_{i+m, j+n},
\end{array}\right.
\end{gathered}
$$

where the variable $h_{i, j}$ denotes the halftone value at position $(i, j)$ of a halftone image $(H)$; variable $e_{i, j}^{2}$ denotes the Mean-Square Error (MSE) between ori $i_{i, j}$ and $\hat{h}_{i, j}$; variable $c_{i, j, o p t}$ denotes the coefficient of the optimized LMS-trained filter $\left(C_{o p t}\right)$, and $\mu$ denotes the adjusting parameter used to control the convergent speed of the LMS optimization procedure. In this work, the variable $\mu$ is set to $10^{-5}$. Some other quality evaluation methods can be found in [10]-[11].

The other performance evaluation is the CDR, which determines the similarity between the original binary watermarks $(W)$ and corresponding decoded watermarks $(D W)$. The CDR is based on the concept of Hamming distance as defined below, 


$$
C D R=\frac{\sum_{i=1}^{P} \sum_{j=1}^{Q}\left(\overline{w_{l, j} \oplus d w_{l, j}}\right)}{P \times Q} \times 100 \%,
$$

where the size of watermarks is $P \times Q$, which is same as original grayscale images. The variables $w_{i, j}$ and $d w_{i, j}$ denote the original watermark $(W)$ and corresponding decoded watermarks $(D W)$, respectively, and the notation $\oplus$ denotes the XOR operation.

\section{Data Hiding with Overall Minimal-Error Searching}

This section describes the proposed OMES encoding scheme, which embeds watermark information into multiple halftone images. The concept is similar to the Secret Sharing Scheme (SSS) [12], and the algorithm is depicted in Fig. 1.

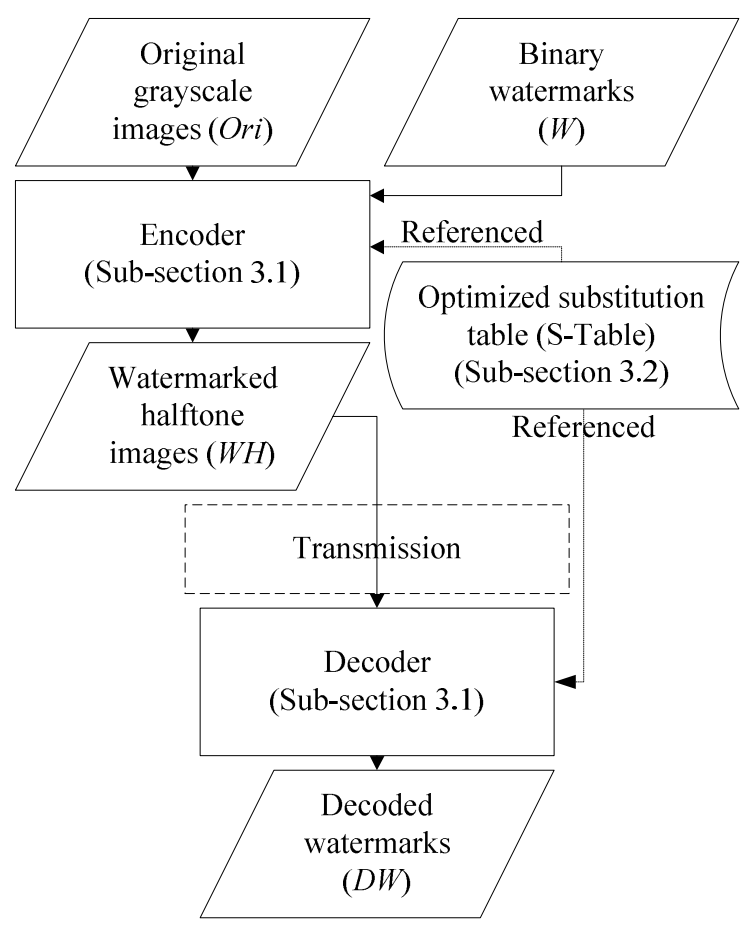

Fig. 1. Flow chat of the proposed algorithm

\subsection{Encoder}

The proposed OMES encoder is illustrated in Fig. 2. The variable or $i_{i, j}^{n}$ denotes the grayscale value at current processing position $(i, j)$ of the $n$th original grayscale image. The variables $h_{i, j}^{n}$ and $w h_{i, j}^{n}$ denote the temporary halftone output $H$ and final watermarked halftone output $W H$ at current processing position $(i, j)$. The blocks with name "Halftoning" represent the typical error diffusion. The function $N(\cdot)$ of Ori denotes 


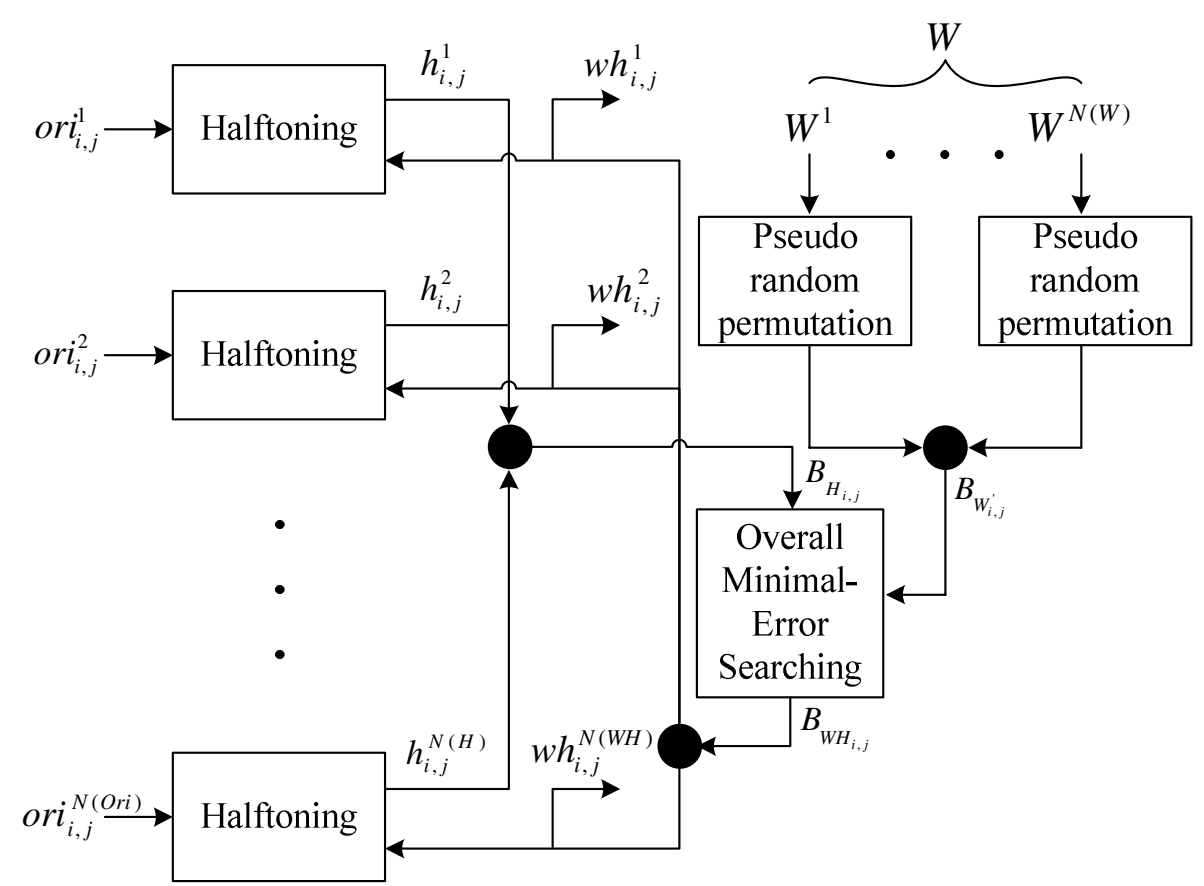

Fig. 2. Proposed Overall Minimal-Error Searching (OMES) encoder algorithm

the number of original grayscale images. In this work, $N($ Ori $)=N(H)=N(W H)$. The variable $W$ denotes watermark set for embedding, and $W^{n}$ denotes the $n$th watermark. After the pre-processing "pseudo random permutation", the new set of watermarks $W^{\prime}$ can be obtained. The variables $B_{H_{i, j}}, B_{W H_{i, j}}$ and $B_{W_{i, j}^{\prime}}$ denote the vector of values $H, W H$, and $W^{\prime}$ at position $(i, j)$, which are with the form of $\left\{h_{i, j}^{1}, h_{i, j}^{2}, \cdots, h_{i, j}^{n}\right\}$, $\left\{w h_{i, j}^{1}, w h_{i, j}^{2}, \cdots, w h_{i, j}^{n}\right\}$, and $\left\{w_{i, j}^{\prime 1}, w_{i, j}^{\prime 2}, \cdots, w_{i, j}^{\prime n}\right\}$.

The OMES observes the temporary halftone output vector and the corresponding permutated halftone values of watermark at same position, and then modifies the output vector according to the S-Table, which will be introduced later. The quantized errors are feedback-diffused to the neighboring pixels of the temporary halftone images (host images) to reduce the damage caused by the binary outputs modification. The $B_{H_{i, j}}$ has many configurations: Assume the number of host images is 2, the corresponding combinations are given in Table I. The number of configurations is $2^{N(H)}$, where $N(H)=2$ in this case. The variable $N(W)$ denotes the number of watermarks, and the number of groups is $2^{N(W)}$. Hence, the Table I(a) can be expressed as Table I(b), where each row represents a group. This table is called the Substitution Table (S-Table), and each number in S-Table is called a state.

Given a specific order of host images, the corresponding $B_{H_{i, j}}$ is first transformed to a decimal value, then this value maps to a corresponding group in S-Table. The mapped group is called original group $\left(G_{O}\right)$. Meanwhile, the vector $B_{W_{i, j}^{\prime}}$ of the watermarks with 


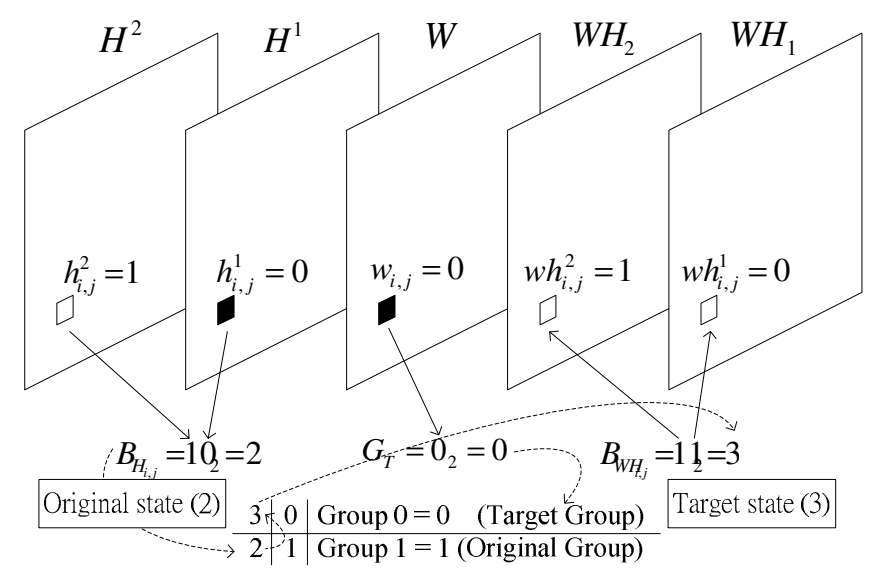

Fig. 3. Example of the $G_{0}$ and $G_{T}$ with two host images and one embedded halftone watermark

the same position as host images is transformed to decimal value, then this value also maps to a corresponding group in S-Table. The watermark mapped group is called the target group $\left(G_{T}\right)$. When $G_{O}=G_{T}$, then let $B_{H_{i, j}}=B_{W H_{i, j}}$; When $G_{O} \neq G_{T}$, then all the states in $G_{T}$ should be used for testing which state causes minimal overall error. Herein, the overall error is defined as the overall quantized error caused by the tested state. The one with the minimal overall error is employed to replace the original state. An example is illustrated in Fig. 3. The procedure of the proposed OMES is organized as below:

$$
\begin{gathered}
\mathbb{S}_{G_{T}}=\left\{s_{G_{T}}^{a} \mid a=1,2, \cdots, 2^{N(H)-N(W)}\right\}, \text { where } 0 \leq s<2^{N(H)}, \\
s_{G_{T}}^{a}=\left\{h_{a, i, j}^{1}, h_{a, i, j}^{2}, \cdots, h_{a, i, j}^{N(H)}\right\}, \text { where } h=\{0,1\}, \\
e_{i, j}^{a}=\sum_{n=1}^{N(H)}\left|v_{i, j}^{n}-h_{a, i, j}^{n}\right|, \\
e_{i, j, \text { min }}^{k}=\min \left(e_{i, j}^{a} \mid a=1,2, \cdots, 2^{N(H)-N(W)}\right),
\end{gathered}
$$

where the vector $\mathbb{S}_{G_{T}}$ denotes the set of states of $G_{T}$ in S-Table, and variable $s$ denotes the state. The variable $a$ denotes the state number in $G_{T}$, and the variable $n$ denotes the host image number. The variable $v_{i, j}^{n}$ denotes the modified grayscale output of the $n$th host image, and the variable $e_{i, j}^{a}$ denotes the difference between the modified grayscale output $v_{i, j}^{n}$ and $a$ th halftone output $h_{a, i, j}^{n}$ in $G_{T}$. The target state $s_{G_{T}}^{k}$ (kth state) is obtained with the steps given in Eqs. (8)-(11), which is able to minimize the overall error and hence maintains the image quality.

However, when the capacity is as high as $50 \%$, a large amount of outputs $w h_{i, j}$ with high quantization error $e_{i, j}$ are forced to be used, which then degrades the image quality. For this, a pre-defined error threshold $e_{t h}$ is set to overcome this problem. The feedback error denotes as following, 


$$
e_{i, j}^{n}=\left\{\begin{array}{c}
e_{t h}, \quad \text { if } v_{i, j}^{n}-h_{k, i, j}^{n}>e_{t h} \\
-e_{t h}, \quad \text { if } v_{i, j}^{n}-h_{k, i, j}^{n}<-e_{t h}, \\
v_{i, j}^{n}-h_{k, i, j}^{n}, \quad \text { Otherwise }
\end{array}\right.
$$

In this work $e_{t h}=127$. In the decoder, we simply need to collect the corresponding halftone values in these watermarked halftone images and form into the decoded vector sets, and then look up the S-Table for decoding.

\subsection{Optimization Procedure for Achieving a Substitution Table (S-Table)}

The watermarked halftone image quality obtained by OMES is determined by the quantity of alternations of halftone value from original state to target state. Theoretically, the halftones created by original state have the best image quality compared to the halftones created by other target states. Nonetheless, the quality between halftones created by target state and original state are demanded to be similar to achieve good embedded image quality. However, when the differences between target and original states have too much discrepancies, the image quality will significant degrade. Based on this, we develop an optimized procedure of constructing S-Table as below.

The 18 different natural grayscale images are employed in the optimization training procedure of S-Table. To generate the training watermarks, a uniform distributed image with grayscale value 128 is adopted to perform three different halftoning approaches: Classical-4 clustered-dot dithering [1], Bayer-5 dispersed-dot dithering [1], and Mese's dot diffusion with class matrix of size $8 \times 8$ [3]. To evaluate the quality of the obtained watermarked halftone image, the average PSNR is calculated. The following steps are developed to provide a fair PSNR distribution across multiple embedded watermarks.

$$
\begin{gathered}
\mathbb{P}=\left\{P S N R_{n} \mid n=1,2, \cdots, N(W H)\right\}, \\
\operatorname{Var}(\mathbb{P})=\sum_{n=1}^{N(I)}\left(P S N R_{n}-\text { Average }(\mathbb{P})\right)^{2} p\left(P S N R_{n}\right), \\
\text { Cost }=\left\{\begin{array}{c}
\sum_{n=1}^{N(I)} P S N R_{n} p\left(P S N R_{n}\right), \text { if } \operatorname{Var}(\mathbb{P})<0.4, \\
0, \quad \text { Otherwise }
\end{array}\right.
\end{gathered}
$$

where the vector $\mathbb{P}$ denotes the set of PSNRs of the watermarked halftone images; the variable $n$ denotes the $n$th watermarked halftone image, and $\operatorname{Var}(\mathbb{P})$ denotes the discrepancies between PSNRs. In this study, the upper bound of $\operatorname{Var}(\mathbb{P})$ is set at 0.4 . The $\mathrm{S}$-Table which achieves the highest cost is employed for applications. The steps of the optimization procedure are organized as below,

Step 1. Given an initial S-Table $(S)$.

Step 2. Suppose the states within S-Table are rearranged as 1-D sequence. Each states $s_{i}$ in the $\mathrm{S}$-Table is successively swapped with one of the other $2^{N(H)}$ states $s_{j}$, where $i \neq j$. 
Step 3. Evaluating the cost of the set of watermarked halftone images using the S-Table obtained from Step 2.

Step 4. The swapped S-table leads to the highest cost, $\max \operatorname{Cost}(S, \operatorname{swapped} S)$, is taken as a new S-Table. Otherwise, the swapped states within S-Table are recovered to their original positions.

Step 5. Another states $s_{i}$ in the S-Table is selected, and then performs Steps 3 and 4.

Step 6. If any swapping cannot improve the cost of the set of watermarked halftone images, the optimization procedure is terminated. Otherwise, Steps 2 to 5 are repeated.

Notably, in order to provide good initial S-Table candidates, 5000 random-generated $\mathrm{S}$-Tables are tested, and the one achieves the highest cost is adopted. Table II shows the final convergent S-Tables for different numbers of host images, which include three configurations: One watermark embedded into two host images, two watermarks embedded into four host images, three watermarks embedded into six host images. All of the configurations can achieve capacity $50 \%$. In this study, no more than eight host images are explored, since it is impractical in applications.

\section{Experimental Result}

Figures 4(d)-(e) show the watermarked halftone images using MEBS [8] with capacity $50 \%$, and Figs. 4(f)-(q) show the watermarked halftone images with the proposed OMES under the same capacity $50 \%$. It is clear that the OMES yields better average image quality than MEBS whether in subjective or objective quality criterion.

An interesting phenomenon is explored as below: When the number of host images is few, such as two, one host image with explicit edge structure or a cluster of bright or dark area, the other watermarked halftone image will suffer from serious interference and then degrades in quality. This problem is raised because there are few selectable states $\left(2^{N(H)-N(W)}\right)$ in target group. To solve this problem, the difference between $N(H)$ and $N(W)$ should be increased. Notably, the ratio between $N(H)$ and $N(W)$ has to be maintained to keep it in the same capacity. For example, $N(H)$ and $N(W)$ are increased from 2 and 1 to 4 and 2, respectively.

The performance comparisons among the various watermarking approaches using 12 test images are depicted in Fig. 5, which include MEBS [8], OMES with two host images and one watermark (EDF 1H2), OMES with four host images and two watermarks (EDF 2H4), OMES with six host images and three watermarks (EDF 3H6), The results consist with the discussions given above. The results show the image quality is improved when the difference between $N(H)$ and $N(W)$ is increased.

Two of the most frequent happened attacks, cropping (tampering) and print-andscan, are involved in the experiments to analyze the robustness performance. The cropping (tampering) rates range from 5\% to $40 \%$. To avoid the cluster losing of information by cropping, the watermark is pseudo-permutated before being embedded. 


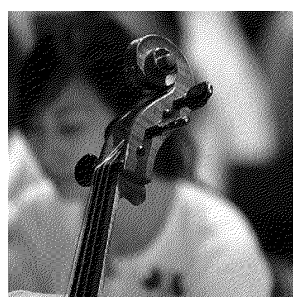

(a)

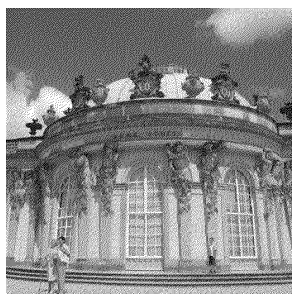

(b)

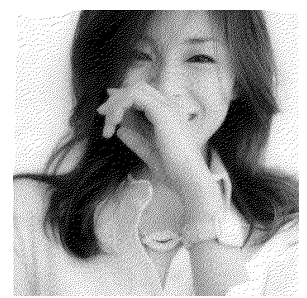

(c)
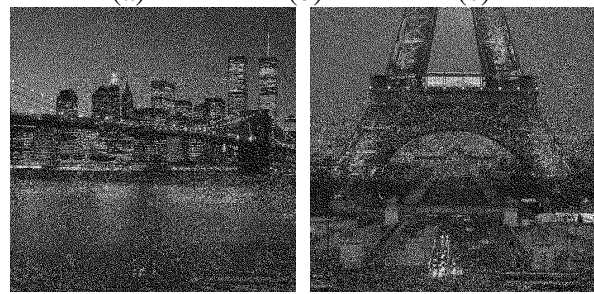

(d) $P S N R=24.5 \mathrm{~dB}$

(e) PSNR=24.4 dB
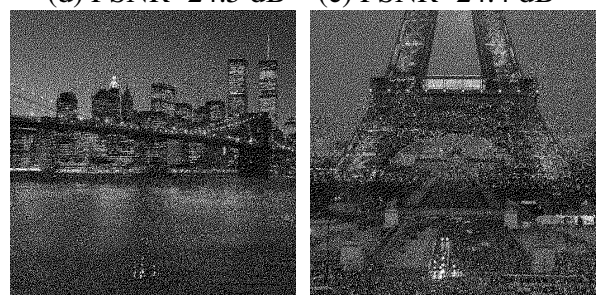

(f) $\mathrm{PSNR}=26.3 \mathrm{~dB}$
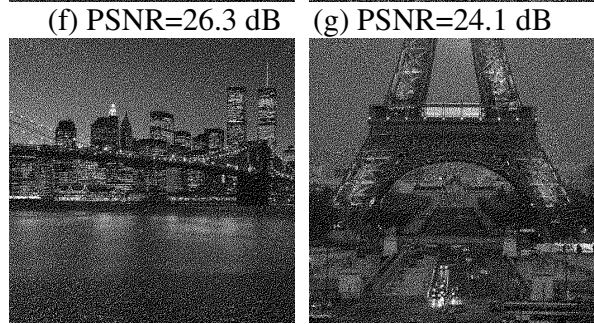

(h) $P S N R=27.9 \mathrm{~dB}$
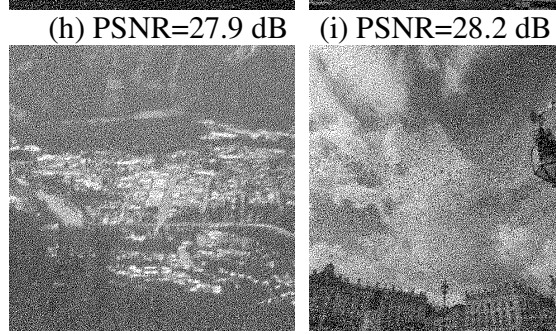

(j) $P S N R=28.9 \mathrm{~dB}$

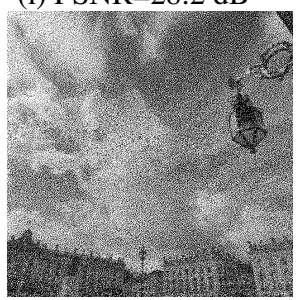

(k) PSNR=25.1 dB

Fig. 4. Watermarked halftone images with the MEBS [8] and proposed OMES, where the S-Table as shown in Table II. (a)-(c) Halftone watermarks. (d)-(e) Two $W H$ s which hide $W$ (a) (MEBS). (f)-(g) Two $W H$ s which hide $W$ (a) (OMES). (h)-(k) Four $W H$ s which hide $W \mathrm{~s}$ (a) and (b) (OMES). (l)-(q) Six WHs which hide W s (a), (b), and (c) (OMES). (all printed at 450 dpi). 


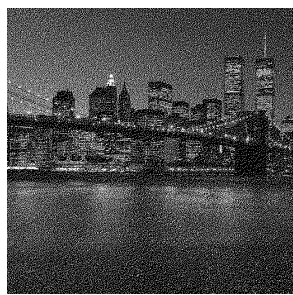

(1) $\mathrm{PSNR}=28.1 \mathrm{~dB}$

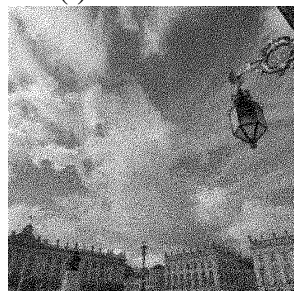

(o) $\mathrm{PSNR}=28.6 \mathrm{~dB}$

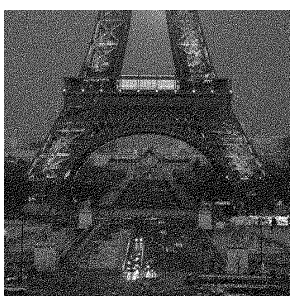

(m) $\mathrm{PSNR}=27.7 \mathrm{~dB}$

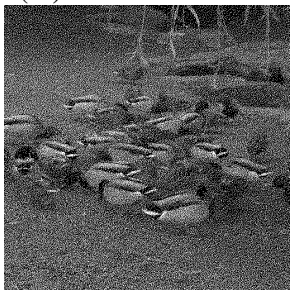

(p) $P S N R=28.2 \mathrm{~dB}$

Fig. 4. (continued)

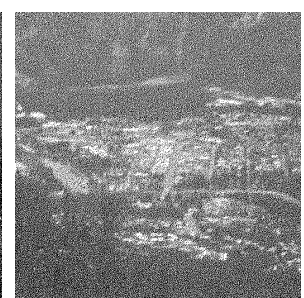

(n) $\mathrm{PSNR}=28.6 \mathrm{~dB}$

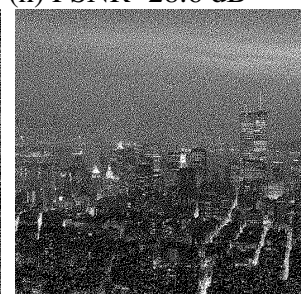

(q) PSNR=27.7 dB

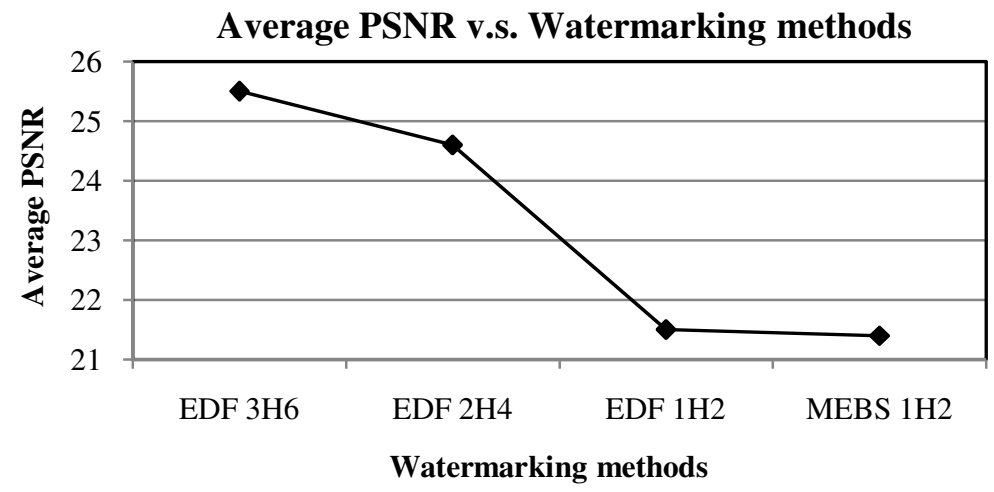

Fig. 5. Performance comparisons between OMES and MEBS [8] watermarking

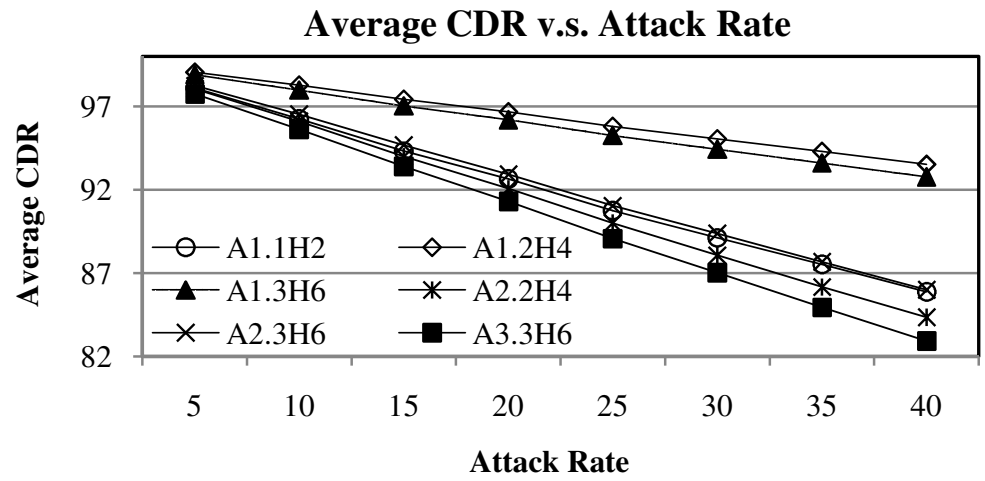

Fig. 6. Cropping attack with cropping rates range from $5 \%$ to $40 \%$ 


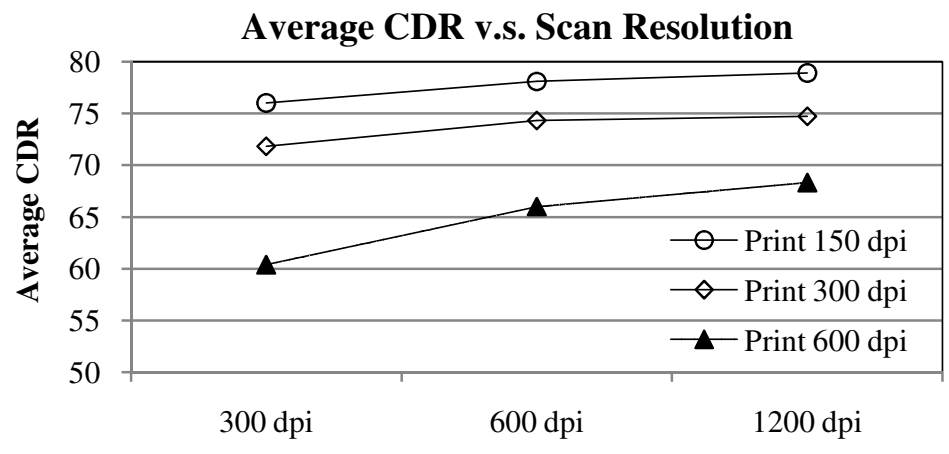

Scan resolution

Fig. 7. Print-and-Scan attack with different configurations of print-and-scan resolutions

Table 1. Substitution table (S-Table): relationships between watermark and the corresponding vector

\begin{tabular}{l|c|c|c|c|c}
$\begin{array}{l}\text { Group \# (Watermark Infor- } \\
\text { mation bit) }\end{array}$ & $\begin{array}{c}\text { Group 0 } \\
\text { (black) }\end{array}$ & $\begin{array}{c}\text { Group 1 } \\
\text { (white) }\end{array}$ & $\begin{array}{c}\text { Group 1 } \\
\text { (white) }\end{array}$ & $\begin{array}{c}\text { Group 0 } \\
\text { (black) }\end{array}$ & $\cdots$ \\
\hline Corresponding decimal value & 0 & 1 & 2 & 3 & $\cdots$ \\
\hline Input Image \#1 & 0 & 1 & 0 & 1 & $\cdots$ \\
\hline Input Image \#2 & 0 & 0 & 1 & 1 & $\cdots$ \\
\hline Input Image \#n & $\vdots$ & $\vdots$ & $\vdots$ & $\vdots$ & $\ddots$
\end{tabular}

(a)

\begin{tabular}{c|c|c}
\multicolumn{2}{c|}{ States } & Group \# \\
\hline 3 & 0 & Group 0 \\
\hline 2 & 1 & Group 1
\end{tabular}

(b)

Table 2. Substitution table trained for 2, 4 and 6 host images with capacity 50\%

\begin{tabular}{c|c|c}
\multicolumn{2}{c|}{ States } & Group \# \\
\hline 3 & 0 & Group 0 \\
\hline 2 & 1 & Group 1
\end{tabular}

(a) For one watermark hiding in two images.

\begin{tabular}{|c|c|c|c|c|}
\hline \multicolumn{4}{|c|}{ States } & Group \# \\
\hline 11 & 9 & 4 & 2 & Group 0 \\
\hline 14 & 5 & 15 & 0 & Group 1 \\
\hline 3 & 13 & 8 & 6 & Group 2 \\
\hline 1 & 12 & 10 & 7 & Group 3 \\
\hline
\end{tabular}

(b) For two watermarks hiding in four images.

\begin{tabular}{c|c|c|c|c|c|c|c|c}
\multicolumn{7}{c}{ States } & Group \# \\
\hline 19 & 36 & 55 & 15 & 13 & 34 & 41 & 24 & Group 0 \\
\hline 60 & 17 & 10 & 54 & 33 & 4 & 59 & 30 & Group 1 \\
\hline 38 & 50 & 12 & 45 & 7 & 53 & 27 & 16 & Group 2 \\
\hline 20 & 62 & 49 & 8 & 56 & 3 & 43 & 29 & Group 3 \\
\hline 39 & 25 & 23 & 18 & 32 & 5 & 61 & 14 & Group 4 \\
\hline 21 & 26 & 6 & 35 & 63 & 40 & 48 & 9 & Group 5 \\
\hline 37 & 46 & 31 & 58 & 44 & 0 & 11 & 22 & Group 6 \\
\hline 51 & 42 & 1 & 2 & 28 & 52 & 47 & 57 & Group 7
\end{tabular}

(c) For three watermarks hiding in six images. 
The embedded images are re-permutated before the decoding is performed. Figure 6 shows the decoded results, where the "A\#" represents the number of attacked watermarked halftone images, and the "types" represents the different host image and watermark configurations with capacity 50\%. Notably, the CDRs are the averaged results of the 12 test images. Finally, multiple configurations of print-and-scan attacks are involved in the experiments, namely printing at 150,300, and $600 \mathrm{dpi}$, and scanning at 300, 600, and 1200 dpi. Here the RGB color image of size $P \times Q$ is used, which is divided into several cells of size $M \times N$. An information bit is embedded in a cell, which means that every vector in cell has to embed the same information bit. The average CDRs with the 12 test images are organized in Fig. 7.

\section{Conclusions}

This work presents a high payload watermarking in multiple halftone images using Overall Minimal-Error Searching (OMES). Employing the concept that a vector can be used to represent an information bit of watermark, the proposed OMES effectively shares a watermark into two halftone images with data capacity 50\%. The image quality can be further improved when the difference between $N(H)$ and $N(W)$ is increased, since more potential selectable state are available. As documented in the experimental results, the image quality of the proposed OMES is significantly superior to the pervious Minimal-Error Bit Searching (MEBS) under the same embedded capacity. Moreover, it can guard against most frequent happened cropping and print-and-scan attacks. Due to the high capacity nature of the proposed method, it is also applicable to secret communication application.

\section{References}

1. Ulichney, R.: Digital Halftoning. MIT Press, Cambridge (1987)

2. Floyd, R.W., Steinberg, L.: An adaptive algorithm for spatial gray scale. In: Proc. SID 75 Dig.: Society for information Display, pp. 36-37 (1975)

3. Mese, M., Vaidyanathan, P.P.: Optimized halftoning using dot diffusion and methods for inverse halftoning. IEEE Trans. Image Processing 9, 691-709 (2000)

4. Hel-Or, H.Z.: Watermarking and copyright labeling of printed images. J. Electron. Imaging 10(3), 794-803 (2001)

5. Fu, M.S., Au, O.C.: Data hiding by smart pair toggling for halftone images. In: Proc. IEEE Int. Conf. Acoustics, Speech and Signal Processing, vol. 4, pp. 2318-2321 (June 2000)

6. Fu, M.S., Au, O.C.: Data hiding watermarking for halftone images. IEEE Trans. Image Processing 11, 477-484 (2002)

7. $\mathrm{Wu}, \mathrm{C} . \mathrm{W}$.: Multimedia data hiding and authentication via halftoning and coordinate projection. Eurasip J. Appl. Signal Processing 2002(2), 143-151 (2002)

8. Pei, S.C., Guo, J.M.: High-capacity data hiding in halftone images using minimal-error bit searching and least-mean square filter. IEEE Trans. Image Processing 15, 1665-1679 (2006) 
9. Mannos, J., Sakrison, D.: The effects of a visual fidelity criterion on the encoding of images. IEEE Trans. Inform. Theory 20, 526-536 (1974)

10. Wang, Z., Bovik, A.C.: A Universal Image quality Index. IEEE Signal Processing Letters 9(3), 81-84 (2002)

11. Damera-Venkata, N., Kite, T.D., Geisler, W.S., Evans, B.L., Bovik, A.C.: Image Quality Assessment Based on a Degradation Model. IEEE Transactions on Image Processing 9(4), 636-650 (2000)

12. Shamir, A.: How to share a secret. Commun. ACM 22, 612-613 (1979) 